\title{
Policies and practices in haemostasis testing among laboratories in Croatia: a survey on behalf of a Working Group for Laboratory Coagulation of the Croatian Society of Medical Biochemistry and Laboratory Medicine
}

\author{
Ana Bronić*1, Desiree Coen Herak², Sandra Margetić1, Marija Milić3,4 \\ ${ }^{1}$ Clinical Institute of Chemistry, Medical School University Hospital "SestreMilosrdnice" Zagreb, Croatia \\ ${ }^{2}$ Department of Laboratory Diagnostics, University Hospital Centre Zagreb, Zagreb, Croatia \\ ${ }^{3}$ Department of Clinical Laboratory Diagnostics, Osijek University Hospital, Osijek, Croatia \\ ${ }^{4}$ Faculty of Medicine, University of Osijek, Osijek, Croatia \\ *Corresponding author: anabronic@yahoo.com
}

\begin{abstract}
Introduction: The objective of this survey was to assess current policies and practice in haemostasis testing among both hospital and outpatient laboratories in Republic of Croatia.

Materials and methods: A questionnaire with seventy questions divided into nine sections was created in May 2015. Participants were asked about their practice related to test request form, sample collection, prothrombin time (PT) and activated partial thromboplastin time assays, other individual haemostasis assays, point-of-care testing (POCT), reporting of coagulation tests results and quality assurance of procedures, the personnel and other laboratory resources, as well as on issues related to education and implementation of additional coagulation assays in their laboratory. The survey was administered and data were collected between June and September 2015.

Results: A total survey response rate was 104/170 (61.2\%). Most respondents were faced with incomplete information on prescribed therapy and diagnosis on the test request or inappropriate samples withdrawn on distant locations, but also do not have protocols for handling samples with high haematocrit values. Reporting of PT-INR and D-dimer results was different between laboratories. Although almost all laboratories developed a critical value reporting system, reporting a value to general practitioners is still a problem. Result on coagulation POCT testing showed that not all devices were supervised by laboratories, which is not in compliance with Croatian Chamber of Medical Biochemistry acts.

Conclusion: Obtained results highlighted areas that need improvement and different practice patterns in particular field of haemostasis testing among laboratories. A harmonization of the overall process of haemostasis testing at national level should be considered and undertaken.
\end{abstract}

Key words: survey; haemostasis; blood coagulation tests

\section{Introduction}

Disorders of haemostasis are associated with substantial morbidity and mortality, and as such are major public health concerns $(1,2)$. In addition to being a challenge for clinicians, majority of haemostasis abnormalities are diagnosed by laboratory examination of the blood coagulation process. Therefore, laboratories have a vital role in diagnosis and management of haemostatic disorders (3).

Modern haemostasis laboratory performs a large number of distinct assays, and the majority of them are screening or specific tests for monitoring anticoagulant therapies (3). Prothrombin time (PT)/International Normalized Ratio (INR) and activated partial thromboplastin time (aPTT) are global clotting assays, frequently employed within most laboratories along with platelet count. Besides, tests such as fibrinogen and D-dimer are mostly used in order to clarify diagnosis. To provide further insight into screening tests abnormalities including both bleeding and thrombotic disorders, specific assays for different coagulation 
components such as factor activity assays, platelet function tests (aggregometry) or thrombophilia assays are used.

Laboratory assessment of the haemostatic system is a major component of clinical management. Due to its complex nature, personnel responsible for test results interpretation, either laboratory scientists or clinicians, are faced with many challenges. Testing process variability can affect result accuracy and consequently cause diagnostic errors with potentially serious effects on patient outcomes $(3,4)$.

In Croatia, haemostasis testing is performed both in transfusion (TL) and medical biochemistry laboratories (MBL) that are supervised by different professional societies. Besides, laboratories operate at different healthcare levels, including those in primary health care (PHC) that mostly perform PT/ INR test only. On the contrary, laboratories within secondary healthcare (SHC) and tertiary healthcare (THC) institutions perform a wide range of different haemostasis assays. The assumption was that substantial variability in laboratory practice and policies could exist. Thus, the objective of this study was to assess the status of current laboratory practice in coagulation testing among both hospital and outpatient laboratories in Croatia.

\section{Materials and methods}

In 2015, the Working Group for Laboratory Coagulation operating within the Committee for scientific and professional development of the Croatian Society of Medical Biochemistry and Laboratory Medicine developed a strategy in order to evaluate the status of current practices of coagulation testing among Croatian laboratories. The first step was to identify the coagulation laboratory network, followed by questionnaire design and implementation of a comprehensive online survey.

To establish the coagulation laboratory network, the last census of health institutions obtained from Ministry of Health and Croatian Institute for Health Insurance was compared with data on laboratories network obtained from the Croatian Chamber of Medical Biochemists (CCMB) and a list of coagulation proficiency testing participants provided by the Croatian Centre for Quality Assessment in Laboratory Medicine.

In May 2015, a questionnaire with seventy questions, divided into nine sections was created. The sections comprised: test request form and sample collection, testing practices for PT, aPTT and other individual haemostasis tests, point-of-care testing (POCT), practice in reporting coagulation test results, practice related to quality assurance (QA) of procedures, to personnel and other laboratory resources, issues related to implementation of additional coagulation tests and education in the laboratory. Detailed instructions for answering the questionnaire were provided by each questionnaire. Questions had multiple answers unless stated otherwise.

This list of 177 laboratories involved in coagulation testing was collated. The questionnaire was sent to their e-mail address, providing the option to each laboratory of responding electronically by email or in written form by regular mail. First reminder was sent to each participant 3 weeks after mailing the survey, and the non-respondents were contacted by phone in order to confirm that the laboratory had received the survey and also to encourage participation. Second reminder was sent to all non-responders after another 3 weeks. From the initial list of laboratory, an automated response on unsuccessful e-mail delivery was recorded from 7 laboratories. As we were unable to get in contact with these, even by phone, and could not get any related coherent information we excluded them from a final number of laboratories dealing with coagulation.

The survey was distributed and data collected between June and September 2015. All collected information were processed in accordance with data confidentiality.

\section{Statistical analysis}

Data for analysis were obtained by counting. Results were presented as numbers or relative frequencies (percentages) of total number of participants or as proportions of observed data alone if the overall number of data was $<100$. Data analyses were performed using Microsoft Excel 2010 
program (Redmond, Washington: Microsoft, 2010 Computer Software).

\section{Results}

\section{Survey response rate}

Of 170 laboratories in the survey, 104 participants returned questionnaires yielding to a total survey response rate of $61.2 \%$. Institutions and the health- care level at which survey participants laboratories operate are presented in Table 1. Considering laboratory type $90.4 \%$ respondents were MBLs, whereas $9.6 \%$ were TL.

\section{Test request form and sample collection}

The majority of respondents (72.1\%) did not have a separate coagulation test request form. Most commonly provided information on test request form

TABLE 1. Institutions and healthcare level of survey participants

\begin{tabular}{ccc}
\hline Institution & Level of the healthcare & $\mathbf{n}(\%)$ \\
\hline Primary health care centre & Primary health care & $47 / 104(45.2)$ \\
General Hospital & Secondary health care & $18 / 104(17.2)$ \\
Special hospital & Secondary health care & $9 / 104(8.7)$ \\
Clinical Hospital Centres & Tertiary health care & $9 / 104(8.7)$ \\
Clinical Hospitals & Tertiary health care & $3 / 104(2.9)$ \\
Clinics & Tertiary health care & $4 / 104(3.9)$ \\
Institute of Transfusion Medicine & NA & $1 / 104(0.9)$ \\
Private institution & NA & $13 / 104(12.5)$ \\
\hline
\end{tabular}

Results are presented as ratio $(\mathrm{n})$ and as percentage/frequency (\%) of total number of respondents (N=104); NA-not applicable.

TABLE 2. Questionnaire results related to test request form and content for coagulation testing

\begin{tabular}{lc}
\hline Questions related to test request form for coagulation testing & n (\%) \\
\hline 1. Laboratory has a separate coagulation test request form with information on the diagnosis and therapy. & $29 / 104(27.9)$ \\
Most common information provided on the coagulation test request form is ${ }^{*}:$ & $24 / 29$ \\
Suspected diagnosis & $16 / 29$ \\
Warfarin therapy & $15 / 29$ \\
Clinical diagnosis & $11 / 29$ \\
Low molecular weight heparin therapy & $10 / 29$ \\
Unfractionated heparin therapy & $10 / 29$ \\
New oral anticoagulants therapy & $9 / 29$ \\
Antiplatelet therapy & $8 / 29$ \\
Use of oral contraceptives &
\end{tabular}

2. Information on therapy is provided independently of having a separate test request form for coagulation $54 / 104$ (51.9) testing.

Information on therapy is provided*:

Sometimes $33 / 54$

Often $18 / 54$

Always $3 / 54$

3. Laboratory is faced with "small and big " coagulogram request 50/104 (48.0)

Results are presented as ratio ( $\mathrm{n}$ ) and as percentage/frequency (\%) of total number of respondents ( $\mathrm{N}=104)$.

*Percentage was not used as number of subjects is < 100; the sum of individual responses can vary since each participant could choose more than one answer. 
TABLE 3. Questionnaire results related to preanalytical issues of coagulation testing

\begin{tabular}{l}
\hline Statement related to preanalytical issues of coagulation testing \\
\hline 1. The laboratory has defined criteria for rejecting coagulation samples. Most common criteria for \\
rejecting coagulation samples stated by participants are*: \\
Haemolysed sample \\
Blood is taken in the tube with wrong anticoagulant \\
Inadequate ratio of blood and anticoagulant (under or overfilled tube >10\%) \\
Clotted sample \\
Test tube is not correctly labelled \\
Mismatch of identification patient's data on the tube and request form \\
Transport of samples is outside of the time frame after blood sampling \\
The sample is not stored on adequate temperature \\
\hline
\end{tabular}

\section{Concentration of sodium citrate used for coagulation testing}

Exclusively 3.2\%

Exclusively 3.8\%

No information provided

3. The laboratory has a defined standard operating procedure for samples with haematocrit values $>0.550 \mathrm{~L} / \mathrm{L}$. $25 / 104(24.0)$

Results are presented as ratio $(n)$ and as percentage/frequency (\%) of total number of respondents $(\mathrm{N}=104)$.

*Percentage was not used as number of subjects is $<100$; the sum of individual responses can vary since each participant could choose more than one answer.

are stated in Table 2. Results related to coagulation tubes in use, criteria for sample rejection and existence of standard procedure for handling samples with haematocrit values $>0.550 \mathrm{~L} / \mathrm{L}$ are presented in Table 3.

\section{Testing practice for $P T$}

All 104 respondents reported performing PT. Information on reagents in use, detection method used for PT testing and PT calibration performance is presented in Table 4. Information of International Sensitivity Index (ISI) value of PT reagents in use was provided by $90.4 \%$ laboratories, ranging from 0.84 to 1.40 (median ISI value 0.99 ). Only $12 / 28$ respondents stated on how they determined mean normal PT (MNPT), mostly using plasma pool from healthy individuals (9/12), and only one participant stated how MNPT was calculated (the arithmetic mean). Determination of MNPT from the calibration curve, as a mean value of reference interval (RI) or by measuring the same sample in duplicate were individual responses. Half of respondents reported INR always as obtained numerical value whereas the other half report INR as a numerical value greater than 4.0 to 13.0 (median
$>$ 6.7). Information on PT result reporting is presented in Table 4.

\section{Testing practice for aPTT}

An estimated $59.6 \%$ of respondents reported aPTT performance. The assay is performed mainly in laboratories not related to PHC institutions (51/57). Results related to reagents in use, aPTT results reporting, therapeutic interval (TI) for monitoring unfractionated heparin (UFH) as well as practice regarding additional actions when a prolonged aPTT result is encountered in patients not taking anticoagulant therapy are presented in Table 5 . Participants were also asked if they routinely initiate further laboratory workup for the presence of lupus anticoagulant (LA), when aPTT correction was not obtained after mixing study. All laboratories that declared to perform aPTT mixing study $(\mathrm{N}=23)$ also reported to initiate further laboratory workup. Nevertheless, $10.6 \%$ of respondents reported to perform LA diagnostic workup profile rather using a lupus-sensitive aPTT reagent (8/11). Furthermore, 15/104 (14.4\%) respondents declared to monitor low molecular-weight heparin (LMWH) therapy. Among them, 7 declared to use anti-Xa 
TABLE 4. Questionnaire results related to prothrombin time testing

\begin{tabular}{|c|c|}
\hline Statement related to $\mathrm{PT}$ testing & n (\%) \\
\hline \multicolumn{2}{|l|}{ 1. Detection method used for PT testing in your laboratory is: } \\
\hline Optical & $50 / 104(48.1)$ \\
\hline Mechanical & 49/104 (47.1) \\
\hline Not specified & $5 / 104(4.8)$ \\
\hline \multicolumn{2}{|l|}{ 2. PT calibration is performed by*: } \\
\hline $\begin{array}{l}\text { PT calibrators } \\
2 \text { calibrators } \\
3 \text { calibrators } \\
4 \text { calibrators } \\
5 \text { calibrators } \\
6 \text { calibrators } \\
\text { precalibrated } \\
\text { no information provided }\end{array}$ & $\begin{array}{c}83 / 104(78.8) \\
1 / 83 \\
9 / 83 \\
1 / 83 \\
20 / 83 \\
39 / 83 \\
1 / 83 \\
12 / 83\end{array}$ \\
\hline Diluting one calibration plasma & 19/104 (18.3) \\
\hline \multicolumn{2}{|l|}{ 3. Source of reagent used for PT testing: } \\
\hline Recombinant & $45 / 104(43.3)$ \\
\hline Innovin (Siemens Healthcare Diagnostics, Marburg, Germany) & $32 / 45$ \\
\hline RecombiPlasTin/RecombiPlastin 2G (Instrumentation Laboratory, Bedford, USA) & $9 / 45$ \\
\hline STA Neoplastine R (Diagnostica Stago, Asnières sur Seine, France) & $4 / 45$ \\
\hline Other (human placenta, rabbit brain) & $59 / 104(56.7)$ \\
\hline Thromborel S (Siemens Healthcare Diagnostics, Marburg, Germany) & $45 / 59$ \\
\hline STA NeoplastineCI Plus (Diagnostica Stago, Asnières sur Seine, France) & $8 / 59$ \\
\hline Hemostat Thromboplastin-SI (Human, Wiesbaden, Germany) & $5 / 59$ \\
\hline STA Neoplastine Cl (Diagnostica Stago, Asnieres sur Seine, France) & $1 / 59$ \\
\hline
\end{tabular}

\section{Reported International Normalized Ratio (INR) value is*:}

Derived from the INR calibration curve

\section{INR is reported on the test report*:}

Always as obtained numerical value

As a numerical value up to a certain critical limit, and greater than the limit

\section{PT results of patients who are not receiving oral anticoagulants are reported in:}

Proportion and INR

Proportion

Percent activity and INR

Proportion and seconds

INR

Percent activity

Proportion and PT ratio 


\section{PT reference interval used in the laboratory is provided by:}

Harmonized reference intervals published by the Croatian

Chamber of Medical Biochemists

$98 / 104(94.2)$

Reference intervals proposed by the manufacturer

$6 / 104(5.8)$

PT - prothrombin time. INR - international normalized ratio. ISI-international sensitivity index. Results are presented as ratio (n) and as percentage/frequency (\%) of total number of respondents $(\mathrm{N}=104)$. ${ }^{*}$ The sum of individual responses can vary from 104 since not all participants responded the question.

TABLE 5. Questionnaire results related to activated partial thromboplastin testing

Statement related to aPTT testing n

\section{Commercial reagent used for APTT testing in laboratory is*:}

Actin FS (Siemens Healthcare Diagnostics, Marburg, Germany) $38 / 62$

Pathromtin SL (Siemens Healthcare Diagnostics, Marburg, Germany) $15 / 62$

Actin FSL (Siemens Healthcare Diagnostics, Marburg, Germany) $5 / 62$

Cephascreen (Diagnostica Stago, Asnières sur Seine, France) $3 / 62$

CK Prest (Diagnostica Stago, Asnières sur Seine, France) $2 / 62$

aPTT Sclavo (Sclavo Diagnostics International, Sovicille, Italy) $1 / 62$

\section{2. aPTT result are reported as:}

Seconds $7 / 62$

Ratio $8 / 62$

Both seconds and ratio $47 / 62$

3. Laboratory has established aPTT therapeutic range for monitoring unfractionated heparin therapy $7 / 62$

4. Actions provided by the respondents when a prolonged aPTT result is encountered in patients not taking anticoagulant therapy

Laboratory do not perform aPTT mixing study $36 / 62$

Laboratory performs aPTT mixing study $\quad 7 / 62$

Laboratory performs aPTT mixing study only in case of an additional request $11 / 62$

Laboratory performs aPTT mixing study if it is ordered as part of lupus anticoagulant testing $5 / 62$

Laboratory is not informed about aPTT mixing study procedure $3 / 62$

aPTT - activated partial thromboplastin time. Results are presented as ratio $(\mathrm{n})$ of total number of participants performing aPTT assay $(\mathrm{N}=62)$.

*The sum of individual responses can vary since each participant could choose more than one answer.

assay exclusively whereas even 8 use aPTT for monitoring LMWH therapy.

\section{Practice related to the performance of other} individual haemostasis tests

The most commonly performed haemostasis tests among respondents are presented in Table 6 . Obtained results showed that even $8.6 \%$ of all respondents i.e., 6 PHC and 3 hospital laboratories, still perform capillary whole blood clotting time (WBCT).
Although all $47 \mathrm{PHC}$ laboratories perform PT, theyrarely perform aPTT (11/47) or fibrinogen (4/47). Specific haemostasis tests such as coagulation factor activities, factor inhibitor assays, or thrombophilia test panel, as well as other assays presented in Table 6 are limited to a small number of mostly specialized coagulation laboratories in SHC and THC institutions.

Almost half of the respondents performed fibrinogen assay and all of them determined fibrinogen activity assay (51/51). Among them, 1/51 laborato- 
TABLE 6. Number of the laboratories that perform particular coagulation tests among the survey respondents

\begin{tabular}{lc}
\hline $\begin{array}{l}\text { Coagulation test performed in the } \\
\text { laboratory is: }\end{array}$ & $\mathbf{n}(\%)$ \\
\hline Prothrombin time (PT) & $104 / 104(100)$ \\
Activated partial thromboplastin time (aPTT) & $62 / 104(59.6)$ \\
Fibrinogen & $51 / 104(49.0)$ \\
D-dimer & $42 / 104(40.4)$ \\
Antithrombin (AT) & $25 / 104(24.0)$ \\
Thrombin time (TT) & $24 / 104(23.1)$ \\
Bleeding time(BT) & $22 / 104(21.2)$ \\
Protein C (PC) & $13 / 104(12.5)$ \\
Coagulation factors & $12 / 104(11.5)$ \\
Platelet aggregation & $12 / 104(11.5)$ \\
Lupus anticoagulant (LA) & $11 / 104(10.6)$ \\
Capillary whole blood clotting time (WBCT) & $10 / 104(9.6)$ \\
Protein S (PS) & $9 / 104(8.7)$ \\
Coagulation factor inhibitors & $8 / 104(7.7)$ \\
Euglobulin clot lysis time & $8 / 104(7.7)$ \\
Plasminogen & $7 / 104(6.7)$ \\
Thrombophilia testing & $7 / 104(6.7)$ \\
Heparin assay (anti-Xa activity assay) & $7 / 104(6.7)$ \\
Fibrin(ogen) degradation products (FDP) & $5 / 104(4.8)$ \\
Activated clotting time (ACT) & $3 / 104(2.9)$ \\
\hline Results are obtained as ratio (n) and as percenta & $1 / 104(1.0)$ \\
\hline
\end{tabular}

Results are obtained as ratio (n) and as percentage/frequency (\%) of total number of respondents $(\mathrm{N}=104)$. The sum of individual responses can vary from 104 since each participant could choose more than one answer.

ry reported also performing antigen assay and 4/51 derived fibrinogen method. The derived fibrinogen results were reported from 2 laboratories, only in patients not taking OAT.

D-dimer methods in use along with measurement units and cut-off values are presented in Table 7. All 42 laboratories used fixed D-dimer cut-off values and the most commonly used values were 0.5 $\mathrm{mg} / \mathrm{L}$ DDU (D-dimer units) and $0.5 \mathrm{mg} / \mathrm{L}$ FEU (fibrinogen equivalent units) (Table 7).

Results related to testing practices for new oral anticoagulants (NOACs) are reported in Table 8.
TABLe 7. Questionnaire results on D-dimer commercial reagents, measurement units and cut-off values.

\begin{tabular}{|c|c|c|c|}
\hline $\begin{array}{l}\text { Commercial } \\
\text { reagent }(\mathrm{N})\end{array}$ & $\begin{array}{l}\text { Measurement } \\
\text { unit }(\mathrm{MU})^{*}\end{array}$ & $\begin{array}{l}\text { Cut-off } \\
\text { value }\end{array}$ & $\begin{array}{c}\text { Combination of } \\
\text { MU and cut-off } \\
\text { value, } N\end{array}$ \\
\hline \multirow{9}{*}{$\begin{array}{l}\text { Siemens } \\
\text { Innovance } \\
(25)\end{array}$} & $\mathrm{mg} / \mathrm{L}$ & 0.5 & 9 \\
\hline & $\mathrm{mg} / \mathrm{L} \mathrm{FEU}$ & 0.5 & 4 \\
\hline & $\mathrm{mg} / \mathrm{L}$ & 0.05 & 1 \\
\hline & $\mathrm{mg} / \mathrm{L} \mathrm{FEU}$ & 4.5 & 1 \\
\hline & $\mathrm{mg} / \mathrm{L} \mathrm{FEU}$ & 0.55 & 2 \\
\hline & $\mu \mathrm{g} / \mathrm{L}$ & 500 & 1 \\
\hline & $\mu \mathrm{g} / \mathrm{L}$ & 550 & 2 \\
\hline & $\mu \mathrm{g} / \mathrm{L}$ & 4280 & 1 \\
\hline & $\mu \mathrm{g} / \mathrm{L}$ FEU & 500 & 4 \\
\hline \multirow{2}{*}{ Vidas (5) } & $\mathrm{mg} / \mathrm{L} \mathrm{FEU}$ & 0.5 & 4 \\
\hline & $\mathrm{ng} / \mathrm{mL}$ FEU & 500 & 1 \\
\hline \multirow{3}{*}{$\begin{array}{l}\text { Roche } \\
\text { Immuno (3) }\end{array}$} & $\mu \mathrm{g} / \mathrm{mL}$ & 5 & 1 \\
\hline & $\mathrm{mg} / \mathrm{L}$ & 0.5 & 1 \\
\hline & $\mathrm{mg} / \mathrm{L} \mathrm{FEU}$ & 0.5 & 1 \\
\hline $\begin{array}{l}\text { Roche } \\
\text { cardiac (3) }\end{array}$ & $\mathrm{mg} / \mathrm{L}$ & 0.5 & 3 \\
\hline Nycocard (2) & $\mathrm{mg} / \mathrm{L}$ & 0.3 & 2 \\
\hline Olympus (2) & $\mathrm{mg} / \mathrm{L}$ & 0.5 & 2 \\
\hline Abbott (1) & $\mathrm{mg} / \mathrm{l}$ & 0.05 & 1 \\
\hline $\begin{array}{l}\text { IL-D-dimer } \\
\text { HS (1) }\end{array}$ & $\mu \mathrm{g} / \mathrm{L}$ & 500 & 1 \\
\hline
\end{tabular}

FEU - fibrinogen equivalent unit. $\mathrm{N}$ - number of laboratories. ${ }^{*}$ Measurement units not assigned with abbreviation FEU are D-dimer units (DDU).

\section{Point of care practices}

An estimated $9.6 \%$ respondents stated that coagulation POCT devices $(\mathrm{N}=18)$ are in use in their laboratories or/and medical facilities (Table 9). Most of POCT users operate within SHC and/or THC facilities $(9 / 10)$. Along with laboratories, clinical departments using coagulation POCT devices are: intensive care units (3/10), Cardiac Surgery Department (2/10), Paediatric Department (2/10) and operating rooms $(1 / 10)$.

Considering PT-INR POCT devices, 4/5 respondents stated its use for monitoring OAT only, and all stated use of the same TI as their central laboratories. One laboratory stated that POCT results ob- 
TABLE 8. Questionnaire results related to testing practices for new oral anticoagulants (NOACs)

\begin{tabular}{lc}
\hline $\begin{array}{l}\text { Statement related to testing practices for } \\
\text { NOACs: }\end{array}$ & $\mathbf{n ( \% )}$ \\
\hline $\begin{array}{l}\text { Laboratory is aware of the issue, but does not } \\
\text { perform it yet }\end{array}$ & $29 / 104(27.9)$ \\
$\begin{array}{l}\text { Laboratory is not informed with the } \\
\text { possibilities of monitoring NOACs }\end{array}$ & $21 / 104(20.2)$ \\
$\begin{array}{l}\text { Laboratory did not declared at all testing } \\
\text { practices for NOACs }\end{array}$ & $20 / 104(19.2)$ \\
$\begin{array}{l}\text { Laboratory has implemented test for } \\
\text { assessing therapy with NOACs }\end{array}$ & $5 / 104(4.8)$ \\
$\begin{array}{l}\text { Laboratory has implemented guidelines for } \\
\text { assessing therapy with NOACs }\end{array}$ & $2 / 104(1.9)$ \\
\hline
\end{tabular}

Results are presented as ratio ( $\mathrm{n}$ ) and as percentage/ frequency (\%) of total number of respondents $(N=104)$. The sum of individual responses can vary from 104 since not all participants responded the question.

TABLE 9. Questionnaire results related to point-of-care testing (POCT) practices

\begin{tabular}{lccc}
\hline \multirow{2}{*}{ POCT device } & \multicolumn{2}{c}{$\begin{array}{c}\text { Device is under } \\
\text { laboratory supervision }\end{array}$} \\
\cline { 2 - 4 } & & yes & no \\
\hline $\begin{array}{l}\text { Prothrombin time - INR } \\
\text { monitoring device }\end{array}$ & 5 & 5 & 0 \\
$\begin{array}{l}\text { Activated clotting time } \\
\text { monitoring device }\end{array}$ & 3 & 1 & 2 \\
$\begin{array}{l}\text { D-dimer device } \\
\text { Thromboleastometer }\end{array}$ & 2 & 2 & 0 \\
Aggregometer & 5 & 2 & 3 \\
Total & 3 & 2 & 1 \\
\hline
\end{tabular}

Results are presented as number of positive (yes) or negative (no) answers. INR - international normalized ratio.

${ }^{*} \mathrm{~N}$-number of point-of-care devices in laboratories or related medical facilities.

tained outside the laboratory are integrated into a central laboratory test report.

Regarding internal quality control (IQC) material, respondents stated equal use of lyophilized plasma (4/10) and/or electronic control (3/10), with frequencies: once per week (3/10), followed by once per month, with reagent lot change or before each analysis. Information on QA and RI for POCT devices not supervised by laboratories was not provided $(6 / 10)$.

Inquiries made by outpatients on how to use POCT PT-INR self-monitoring devices was noted by $15.4 \%$ laboratories. Moreover, one laboratory recorded a complaint on unacceptability of analysing PT-INR exclusively from venous plasma samples, from a patient living abroad who needed the service.

\section{Practice related to reporting coagulation test results}

Results related to test report content are presented in Table 10. When considering critical values, $55.8 \%$ laboratories reported analysis repetition along with its documentation. Obtained critical values are reported to the physician by phone with $(66.3 \%)$ or without $(24.0 \%)$ documentation of the relevant reported information. Most respondents (92.3\%) used CCMB recommendations, whereas two laboratories used other documents or expert opinions.

TABLE 10. Questionnaire results related to laboratory report content

\begin{tabular}{lc}
\hline Coagulation laboratory report contains & $\mathbf{n}(\%)$ \\
\hline Measurement units & $97 / 104(93.3)$ \\
Sample remarks & $90 / 104(86.5)$ \\
Reference intervals & $95 / 104(91.3)$ \\
Therapeutic intervals & $74 / 104(71.2)$ \\
Notice on inability to perform certain analysis & $53 / 104(51.0)$ \\
Recommendations for further testing & $20 / 104(19.2)$ \\
Result interpretation & $10 / 104(9.6)$ \\
Treatment recommendations & $6 / 104(5.8)$ \\
Drug interactions & $5 / 104(4.8)$ \\
Suggested diagnosis & $5 / 104(4.8)$ \\
Testing methodology/reagents & $4 / 104(3.9)$ \\
Recommendations for family members & $2 / 104(1.9)$ \\
\hline
\end{tabular}

Results are presented as ratio ( $\mathrm{n}$ ) and as percentage/ frequency (\%) of total number of respondents $(\mathrm{N}=104)$. The sum of individual responses can vary from 104 since each participant could choose more than one answer. 
TABLE 11. Questionnaire results related to quality assurance (QA) of procedures

\begin{tabular}{lc}
\hline Quality of procedures is mainly assured by: & $\mathbf{n}(\%)$ \\
\hline Periodic calibration of all instruments & $87 / 104(83.7)$ \\
$\begin{array}{l}\text { Outputting results along with reference intervals } \\
\text { Comparing patients results with previous }\end{array}$ & $75 / 104(72.1)$ \\
$\begin{array}{l}\text { results (delta check) } \\
\text { Validation or verification of a new method }\end{array}$ & $68 / 104(70.2)$ \\
$\begin{array}{l}\text { when introducing it in routine practice } \\
\text { QC analysis in duplicate }\end{array}$ & $35 / 104(33.7)$ \\
$\begin{array}{l}\text { Patient analysis in duplicate } \\
\text { Periodical evaluation of critical values }\end{array}$ & $26 / 104(25.0)$ \\
$\begin{array}{l}\text { Consensus with clinicians regarding critical values } \\
\text { Checking the platelet number after sample }\end{array}$ & 5/10/104 (14.4) \\
centrifugation & \\
\hline
\end{tabular}

QC - quality control. Results are presented as ratio (n) and as percentage/frequency (\%) of total number of respondents $(\mathrm{N}=104)$. The sum of individual responses can vary from 104 since each participant could choose more than one answer.
Practice related to quality assurance of procedures

Results related to procedures used in order to assure quality of testing are presented in Table 11. Practice related to IQC procedures is presented in Table 12.

\section{Practice related to personnel and other laboratory resources}

Results on emergency testing service availability, responsibilities, competences, consulting and recommendations in use, as well as practice problem solving are presented in Table 13. In 51.9\% respondents, available emergency test panel was created on basis of CCMB policy documents.

\section{Implementation of additional coagulation tests and further education \\ An estimated $61.5 \%$ of respondents were satisfied with a currently performed coagulation panel in}

TABLE 12. Questionnaire results related to internal quality control (IQC) procedures

\begin{tabular}{lc}
\hline \multicolumn{1}{c}{ Internal quality control (IQC) procedures } & n (\%) \\
\hline 1. Daily IQC check by commercial control plasma is performed & \\
Simultaneously at normal and pathological levels & $80 / 104(76.9)$ \\
By intermittent use of normal and pathological levels alternately day by day & $14 / 104(13.5)$ \\
By use of one control level exclusively & $7 / 104(6.7)$ \\
\hline 2. Frequency of IQC check* & $44 / 104(42.3)$ \\
Before each batch of samples & $38 / 104(36.5)$ \\
Every 24 hours (at the beginning of each day) & $10 / 104(9.6)$ \\
Every 12 hours (at the beginning of each shift) & $6 / 104(5.8)$ \\
Every 8 hours & $13 / 104(12.5)$ \\
\hline 3. Daily IQC check by patient plasma*t & $12 / 13$ \\
Exclusively as daily control following IQC with commercial plasma prior to sample series of PT, aPTT and fibrinogen & $2 / 13$ \\
\hline For inter/intralaboratory agreement between different analysers of routine coagulation tests
\end{tabular}

\section{Reported storage conditions of patient plasma used for IQC*†}

Use of fresh samples

- Maximum frozen time period is 24 hours

- Maximum frozen time period is 72 hours

- Double plasma centrifugation prior to freezing

- Not specified

PT - prothrombin time. aPTT - activated partial thromboplastin time. Results are presented as ratio (n) and as percentage/ frequency (\%) of total number of respondents $(\mathrm{N}=104)$.

${ }^{*}$ The sum of individual responses can vary since each participant could choose more than one answer.

tPercentage was not used if number of subjects is $<100$. 
TABLE 13. Questionnaire results related to personnel and other laboratory resources

Statement n (\%)

\section{Availability of emergency test service*}

8 hours through working days

2. Responsible for implementation of coagulation testing in laboratory is ${ }^{\dagger}$ :

Medical biochemists, specialist

Medical biochemists, master

Medical doctor, specialists in transfusion medicine

Medical doctor, other speciality

\section{Staff competence is assured by':}

Successful QC performance and if needed, documenting corrective actions

Direct observation of the task

Study of available literature

Analysing unknown samples

\section{Laboratory use recommendations provided by:}

CCMB

\section{Laboratory provide consulting service:}

Yes

No

Not stated

\section{Comments on coagulation test report are provided by ${ }^{\dagger}$}

Medical biochemists, specialist

Medical biochemists, master

Medical doctor, specialists in transfusion medicine

7. Laboratory operates within institution that have consultant haemostasis disorder expert(s)

$23 / 104(22.1)$

8. Laboratory operates within institution that has specialized anticoagulation clinic $18 / 104(17.3)$

9. Laboratory operates within institution that has department for bleeding disorder $17 / 104(16.3)$

\section{Daily practice problems are solving by*}

Personal contact

\section{Laboratory is aware of availability of other coagulation assays not available in their laboratory*}

Fully

12. Laboratory has defined protocol for sending samples to other institutions ${ }^{\dagger}$ :

Yes

No

QC - quality control. CCMB - Croatian Chamber of Medical Biochemists. Results are presented as ratio ( $\mathrm{n}$ ) and as percentage/ frequency (\%) of total number of respondents $(\mathrm{N}=104)$.

*The sum of individual responses can vary from 104 since not all participants responded the question.

'The sum of individual responses can vary from 104 since each participant could choose more than one answer. 
their laboratories with respect to institution needs. A need to introduce additional coagulation test(s) was noted in 38/104 (36.5\%) respondents. Among respondents from $\mathrm{PHC}$ need to introduce $\mathrm{PPTT}$ (13/15) fibrinogen (5/15) and D-dimers (5/15) was noted. Respondents belonging to SHC and THC mostly stated need to introduce certain specialized assays, such as NOACs determination (7/23), tromboelastometry (4/23), platelet aggregation (3/23), anti-Xa activity (3/23), LA (3/23), thrombophilia panel (3/23), AT (3/23), PC (3/23), PS (3/23), heparin induced thrombocytopenia (HIT) (2/23),
TT (2/23), coagulation factors VIII (FVIII) $(2 / 23)$ and IX (FIX) (1/23), antiplatelet antibodies (1/23).

Finally, participants were asked to give opinions on the most important problem they encounter in daily practice related to haemostasis testing. Of all received responses (42/104), sample quality was stated as the most common problem (17/42), followed by lack of appropriate data on prescribed therapy and diagnosis on test request (10/42) and incorrect test requesting (4/42). Other problems were less common and are presented in Table 14.

TABLE 14. Questionnaire results related to most common problems encountered in daily practice

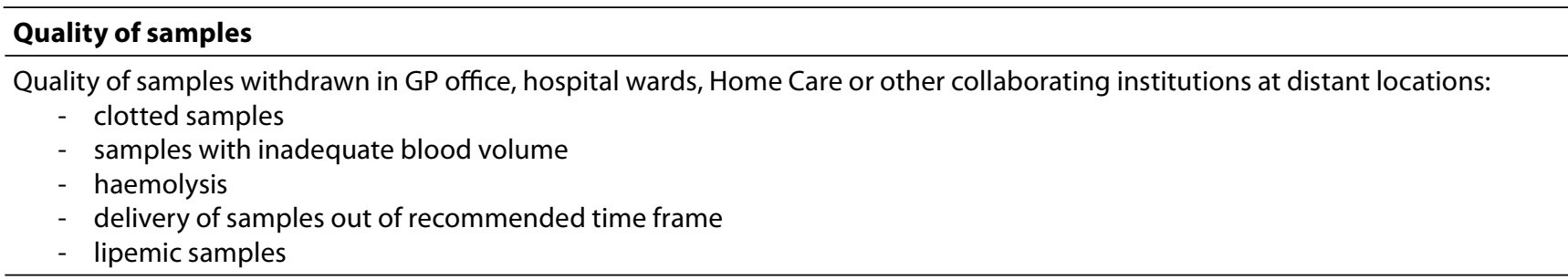

\section{Therapy and diagnosis}

Lack of data on diagnosis and/or prescribed anticoagulant therapy

- on the test request from clinicians

- on the test request shipped via CEZIH's

Test results are not in concordance to therapy stated on the test request

Problems related to knowledge on anticoagulant therapy

- impact of therapy on certain coagulation assays is not clear, especially for thrombophilia screening test panel

The purpose of INR is not clear

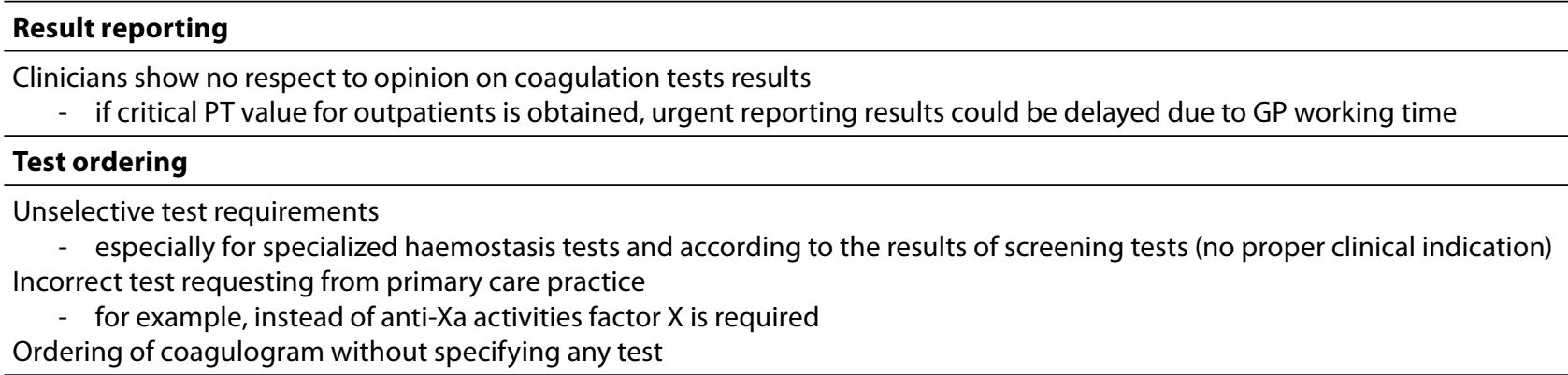

\section{Reagent quality}

Rationalization on reagent consumption

Stability of reagents and control samples if unpredictable low number of request occurred

\section{Harmonization}

Test results are not in concordance (harmonized) to the results obtained in other institutions possibly due to different methodology

\section{Analyzer issues}

Inappropriate space for coagulation analysers and coagulation test performance

Analysers with the optical measurement principle

- difficulties in obtaining results when plasma turbidity occurred due to lipemia or other reasons

CEZIH-central health information system in Croatia. GP-general practice. INR-international normalized ratio. PT-prothrombin time. 


\section{Discussion}

The purpose of this survey was to find out how Croatian hospital and outpatients laboratories handle coagulation testing issues. Obtained results confirmed the assumption that different practice patterns exist among laboratories, pointing to a need for harmonization of the overall haemostasis testing process.

This survey showed that majority of laboratories do not have separate coagulation test requests, which is certainly not surprising due to wide implementation of information systems in medical facilities through the country. However, the main problem that respondents encountered was not related to request type, but to lack of information on diagnosis, prescribed anticoagulation therapy, or too general requests such as "small and big coagulogram". Although definition on such coagulograms doesn't exist, by a tradition the "small" coagulogram is considered to comprise PT and APTT, whereas addition of TT, fibrinogen and/or coagulation factors assays is considered as "big" coagulogram. Looking for supplementary information is time-consuming and could also result in additional costs due to unnecessary (re)testing. More comprehensive collaboration between general practitioners (GPs) and hospital specialists that send laboratory requests should be considered.

Almost all respondents defined criteria for rejection of inappropriate coagulation samples, leading to a better overall response rate compared to study of Shanganian et al. However, poorer results were obtained for criteria such as outside timeframe transport of samples and non-adequate temperature storage (5). Sample quality is influenced by processing and storage and any delay in transportation could lead to prolongation of screening times or factor activity loss $(4,6)$. Therefore these criteria should be implemented in the existent procedures.

This survey revealed that some laboratories still use $3.8 \%$ citrate tubes which are not in compliance with current recommendations (7). The results from these samples may be overestimated especially when inappropriate sample volume is obtained or if $\mathrm{RI}$ based on results derived from 3.2\% citrated tubes are applied (4-7). Furthermore, most respondents did not define a standard procedure for handling samples with haematocrit values $>0.550 \mathrm{~L} / \mathrm{L}$, indicating that they were not fully aware that failure of adjusting citrate in such samples can lead to erroneous results as citrate excess could increase measured clotting times (8).

Our findings related to coagulation tests performance were similar to other international surveys which also documented PT and aPTT as the most commonly performed coagulation assays (5).

PT expressed as INR should be used for OAT monitoring and reliable INR determination is mandatory. As variable INR values in the same plasma sample could be obtained on different coagulometers, ISI calibration of all routinely used reagents is very important (9). This survey showed that most respondents use PT reagents derived from human placenta and rabbit brain although recombinant tissue factor reagents have better sensitivity to factor deficiencies and plasma samples from patients treated with OAT (10). Respondents mostly determine INR values from the specific INR calibration curve, which is in concordance with currently approved CLSI guidelines (11). General consensus regarding optimal ISI value is still not agreed, butcertain societies recommend ISI values $<1.20$ due to precision improvement in INR determination $(10,12)$. Declared ISI values of reagents currently in use among our respondents were even lower (0.84-1.40; median 0.99) compared to previously published surveys, showing that Croatian laboratories follow recommendation provided in the document Harmonized RI published by $\operatorname{CCMB}(5,13-15)$.

Although almost all respondents used harmonized PT RI provided by CCMB (15), unexpected findings were obtained on result reporting for patients not receiving OAT, as half respondents stated to report INR along with PT proportion. Only $22.1 \%$ respondents report it just as proportion. The main reason for non-selective INR reporting could be incomplete information on therapy and diagnosis, as survey results showed that this information are usually not provided on test request form. Therefore, an obvious need for urgent im- 
provement in obtaining relevant information on anticoagulant therapy should be considered as an important prerequisite for adequate PT result reporting in patients with and without OAT.

Half of the respondents report INR always as obtained numerical value whereas the other half report INR as numerical value up to a certain critical limit, and greater than the limit afterwards. There were 16 limit values ranging from 4.0 to 13.0 (median 6.7) reported, similar to a study of Howanitz et al. who reported 18 different critical value limits ranging from 2.6 - 10.0 (median 4.5) (14). As reporting of obtained critical INR value could be confusing to physicians, we could agree with Howanitz et al. that median value of all laboratories should be considered for harmonizing INR reporting $(14,16)$.

This survey showed that aPTT is performed in much lower proportion than in other national surveys including hospital laboratories only. However, when results were restricted to $\mathrm{SHC}$ and THC laboratories almost all of them perform aPTT, similar to another national survey (98.6\%) evaluating only hospital laboratories (5).

This survey also revealed that not all laboratories performing aPTT, report results both as seconds and ratio. Although reporting of aPTT results in seconds only is useful for patient's management, it can lead to inappropriate result comparison between different laboratories. As aPTT reagents differ considerably in their composition variability i.e., phospholipids and activators, leading to different UFH and LA sensitivities, aPTT ratio, a calculated parameter with unique $\mathrm{RI}$ independent of the reagent used should also be stated on the test report (15).

Our results related to aPTT performing practices in patients treated with UFH are quite different from others. In a Canadian survey even 66\% laboratories had established individual aPTT TI and 47\% laboratories verified this range with heparinised samples (17), similar to an American survey showing that $58.8 \%$ hospital laboratories use aPTT TI for UFH (5). On the contrary, only $7 / 62$ of our respondents have a defined aPTT TI for UFH monitoring. However, different investigations demonstrated that standardization between laboratories using
aPTT for monitoring UFH therapy is quite poor. Each laboratory should determine their aPTT reagent sensitivity to UFH and establish local heparin TI for every new reagent lot, reagent type or instrument which is very complicated in routine (11, 18, 19).

LMWH use has at least partly suppressed many aPTT testing issues in the context of heparin monitoring and replaced UFH as the preferred option in many clinical situations (19). As expected, survey respondents from Clinical Hospital Centres and Clinical Hospitals declared to use exclusively antiXa assay, while smaller hospital laboratories use aPTT for monitoring LMWH therapy. Valuable guidelines recommend the chromogenic anti-Xa assay as the only method for LMWH monitoring. LMWHs does not exhibit an adequate impact on aPTT $(18,20)$, since their anticoagulant action is mostly achieved by inhibiting activated FX rather than thrombin or activated FIX. Therefore, use of an inappropriate aPTT assay for LMWH monitoring could potentially have an adverse clinical outcome.

Mixing studies are often performed in order to determine whether PT or aPTT prolongation is due to factor deficiency or to presence of a circulating specific or nonspecific inhibitor such as LA (21). This survey showed that more than half of laboratories performing aPTT do not offer aPTT mixing study or offer it occasionally when an additional order is placed for patients with a prolonged aPTT not receiving anticoagulant therapy and/or as a part of LA workup testing. Recent LA testing guidelines from the International Society on Thrombosis and Haemostasis (ISTH) stress that mixing test should be performed when prolonged aPTT is found (22). Furthermore, mixing test should always be performed with LA-sensitive reagent, as false negative results may be obtained using relatively LA-insensitive reagents. Majority of our survey respondents performing LA testing declared to use LA-sensitive reagents that is in concordance with current recommendations (22).

Several laboratories stated WBCT performance, despite the well-known fact that WBCT is an outdated assay, replaced for years with PT and APTT 
as part of patient's preoperative evaluation. Besides, bleeding time (BT) is still used as a screening test for the diagnosis of some inherited platelet function disorders. As application of both BT and WBCT in preoperative analysis is highly questionable due to low sensitivity and low negative predictive value, their use in routine preoperative evaluation is not recommended (23), and should undoubtedly be replaced with platelet count, PT and aPTT.

Almost half of survey respondents declared to perform the fibrinogen assay, all by using the functional activity assay known as Clauss fibrinogen assay. Furthermore, only minor number of respondent declared to perform fibrinogen antigen assay and derived fibrinogen method. The use of fibrinogen antigen assays that measure protein concentration is limited to the evaluation of hereditary dysfibrinogenemias where there is a discrepancy between functional activity and antigen levels (24). Further, literature data have shown great controversy regarding suitability of derived fibrinogen method for clinical use due to method inaccuracy compared to the Clauss method (25-28). Therefore Clauss functional fibrinogen assay appears to be a method of choice for general use in clinical laboratories (29).

Our survey results showed considerable variability in reporting D-dimer results, either in FEU or DDU units. It must be emphasized that D-dimer values expressed in FEU are approximately two-fold higher than those expressed in DDU meaning that 1.0 $\mathrm{mg} / \mathrm{L}$ FEU corresponds to $0.5 \mathrm{mg} / \mathrm{L}$ DDU. Moreover, we identified a number of different D-dimer measurement units currently in use that could have a confusing effect on clinicians, when receiving different reports from neighbour's laboratories. For example, the value of $0.5 \mu \mathrm{g} / \mathrm{mL}$ corresponds to $500 \mu \mathrm{g} / \mathrm{L}$ or $0.5 \mathrm{mg} / \mathrm{L}$, which is same as $500 \mathrm{ng} / \mathrm{mL}$. D-dimer cut-off values are established by reviewing high risk candidates undergoing objective testing and test sensitivity and specificity should be optimized to make it clinically useful. Thus, each laboratory should establish its own cutoff value and not rely on manufacturer's recommended value or validation in another laboratory. As the main criteria for establishing the cut-off is a high negative predictive value, it should be always kept in mind that the value of D-dimer testing is the absence of elevated D-dimer. The most commonly used fixed D-dimer cut-off values in our survey were $0.5 \mathrm{mg} / \mathrm{L}$ and $0.5 \mathrm{mg} / \mathrm{L} \mathrm{FEU}$.

D-dimer levels rise considerably with age and the use of age-adjusted cut-off values is increasingly discussed as improvement of specificity without too much compromising sensitivity (30). In this survey, no laboratory appeared to use age-adjusted cut-off values. Recent international survey of Lippi et al showed that $93 \%$ of laboratories use a fixed D-dimer cut-off value, whereas only $7 \%$ laboratories use age adjusted cut-offs (31). Evidences obtained in both surveys underline the need for further standardization in D-dimer result reporting. In general, D-dimer testing lacks standardization all over the world since there is still no standardized measurement unit, unique monoclonal antibody and calibrator $(32,33)$. Thus results, RI and clinical cut-off values cannot be extrapolated between methods. Obviously, an effort should be directed towards harmonization of available commercial D-dimer methods.

Furthermore, we examined current status related to guidelines for assessing therapy with NOACs. NOACs are considered to be more convenient for use compared to conventional anticoagulants, since dose adjustment and monitoring are not required for the majority of patients. However, one of the challenges of using these drugs is that they have a different impact on routine coagulation test results, such as PT or APTT which are certainly not suitable for their monitoring. Interpretation of routine global assays may be a problem as abnormal results may be misinterpreted by non-expert clinicians. The biggest educational challenge will not be with clinicians who prescribe and manage anticoagulant therapy, but with those not routinely involved in anticoagulant care, who will encounter an increasing number of patients incidentally taking NOACs (34). Although rarely, in several circumstances such as overdose or in patients with impaired renal function, assessment of the NOACs anticoagulant effect will be required (35). At present, appropriate tests for NOACs are not available in most laboratories, and it is unlikely that many 
laboratories could implement these assays in routine practice. Results of our survey found that almost all respondents did not implemented guidelines for assessing NOACs therapy at present. This finding confirms the fact that there is a clear need for education and implementation guidelines related to the laboratory role of treatment with NOACs.

According to survey results, coagulation POCT devices were in use in $9.6 \%$ of medical facilities only. However, the most important finding was that POCT devices such as tromboelastometry (TEM) or activated clotting time (ACT) mostly are not under the supervision of MBLs, which is not in compliance to CCMB recommendations (36). Similar to CCMB, British Committee for Standards in Haematology guidelines clearly state: "the head of the clinical laboratory or a POCT coordinator must take responsibility for all aspects of the POC testing, including QC and training" $(37,38)$. As aggregometry and TEM are becoming a standard diagnostic tool in perioperative and postoperative acute bleeding management, an increased usage in certain departments could be expected. Therefore, an obvious need for urgent collaboration on POCT issue between laboratories and clinicians should be considered.

It is surprising that only 16 laboratories received a request from patients related to PT-INR self-monitoring devices. These devices are commonly used in some western countries and recent investigations showed that they are an acceptable alternative to laboratory INR monitoring (39). They are certainly less time consuming for patients and several reviews showed better mean time in therapeutic range for self-monitoring patients (40).

When considering IQC performance, most respondents meet recommended minimum criteria (41), but surprisingly, $12.5 \%$ of respondents reported use of patient plasma as an IQC material in conjunction with commercial controls (41). Patient plasma is an important factor in analyzer comparison, but due to many circumstances, e.g., stability of factor activities in fresh plasma, influence of storage temperature and processing time on the results we believe that it would not be appropriate to use it exclusively as IQC material (6).
This survey showed that implementation of coagulation testing is a MBs responsibility both in MBL and $T L$; although in $T L$ supervision is done by transfusiologist. This is in concordance with a formal organisation in Croatia and quite different compared to EU or U.S. laboratories where haematology specialists are mostly involved in implementation of coagulation testing $(5,42)$.

Our survey showed that most laboratories are compliant with CCMB recommendations for test reporting (43). Result interpretation or recommendation for further testing is implemented in small number of specialized coagulation laboratories. Further, consulting service related to coagulation testing are provided by only $36.5 \%$ of laboratories. This is partly due to the traditional view that consultancy should not be provided by laboratory staff and the fact that therapy dose change is not within a MB competence. Furthermore, notes on testing methodology is provided by few laboratories only, mainly when reporting $\mathrm{D}$-dimer results. However, good knowledge of all circumstances surrounding the performed test is precondition for proper information to be given on test report. As our survey showed that medical doctors are rarely involved in coagulation laboratory work, more collaboration with clinicians and development of appropriate policies in post analytical phase of coagulation testing must be considered $(5,43)$.

This survey showed that availability of emergency coagulation testing depends on the level of the health care service, i.e. 8 hours through working days in PHC and 24 hours 7 days a week in smaller number of SHC/THC laboratories. The Emergency test panel is in compliance with CCMB recommendations in most laboratories that adhere to the CCMB document on critical values $(17,43)$. Most laboratories report critical values to physicians by phone, although not all of them regularly document the call, which is similar to results from other surveys $(5,16,17)$. The most common problem on critical values reporting, encountered by participants was critical result reporting to GPs due to shift work. As an established system of critical values reporting is an important laboratory quality indicator, design of comprehensive guidance in re- 
porting coagulation critical values should be considered.

Although most respondents stated that they were satisfied with the currently performed coagulation panel with respect to their medical facility needs, $36.5 \%$ of laboratories, stated the need to introduce additional tests. It is not surprising as most survey respondents were $\mathrm{PHC}$ laboratories that at present perform only PT.

The main problems encountered in daily practice were mostly related to preanalytical issues, especially to inappropriate samples withdrawn at distant locations, as well as communication and collaboration between GPs, clinicians and laboratory staff related to anticoagulation therapy. Problem solving through personal contact and/or reviewing available literature are equally important. More than half of respondents are not aware of the availability of the certain coagulation tests in other facilities, and most laboratories do not have a defined protocol for sending coagulation samples to other medical facilities. A national referral policy for specialist's investigations in bleeding and coagulation disorders should be set up in Croatia.

In summary, we believe that this survey has covered the most important aspects in the field of

\section{References}

1. ISTH Steering Committee for World Thrombosis Day. Thrombosis: a major contributor to the global disease burden. J Thromb Haemost 2014;12:1580-90. https://doi. org/10.1111/jth.12698.

2. Shapiro AD, Soucie JM, Peyvandi F, Aschman DJ, DiMichele DM. Knowledge and therapeutic gaps: a public health problem in the rare coagulation disorders population. Am J Prevent Med 2011;41:S324-S31. https://doi.org/10.1016/j. amepre.2011.09.021.

3. Harris NS, Bazydlo LAL, Winter WE. A Primer on Haemostasis for Clinical Chemists. Clinical Laboratory News 2012. Available at: https://www.aacc.org/publications/cln/articles/2012/January/coagulation-tests. Accessed March 20th 2016.

4. Favaloro EJ. Funk DMA, Lippi G. Pre-analytical variables in coagulation testing associated with diagnostic errors in hemostasis. Lab Med 2012; 43:1-10. https://doi.org/10.1309/ LM749BQETKYPYPVM. laboratory coagulation. However, although questionnaire responses were not obtained from all laboratories performing coagulation assays, almost all MBL from general hospitals, TLs and special laboratories for haematology and coagulation providing higher number of coagulation tests to patients across the country, responded the survey. Therefore, the response rate for particular tests and aspects of testing is certainly higher than the overall response rate. The results highlight different practice policies in particular areas of haemostasis testing between Croatian laboratories and many important fields need improvement. Information gathered in this survey will be the starting point for the development of national guidelines and recommendations on coagulation sample handling and global coagulation assays testing as well as corresponding result reporting.

\section{Acknowledgments}

We thank to all medical biochemistry and transfusion laboratories in Croatia that participated in the survey.

\section{Potential conflict of interest}

None declared.

5. Shahangian S, Stankovic AK, Lubin IM, Handsfield JH, White MD. Results of a survey of hospital coagulation laboratories in the United States, 2001. Arch Pathol Lab Med 2005; 129:47-60.

6. Clinical Laboratory Standards Institute (CLSI). Collection, Transport, and Processing of Blood Specimens for Testing Plasma-Based Coagulation Assays and Molecular Hemostasis Assays; Approved Guideline-Fifth Ed. CLSI document H21-A5. Wayne, PA: CLSI; 2008.

7. Adcock DM, Kressin DC, Marlar RA. Effect of $3.2 \%$ vs $3.8 \%$ sodium citrate concentration on routine coagulation testing. Am J Clin Pathol 1997;107:105-10. https://doi.org/10.1093/ ajcp/107.1.105.

8. Marlar RA, Potts MR,Marlar AA. Effect on routine and special coagulation testing values of citrate anticoagulant adjustment in patients with high hematocrit values. Am J Pathol 2006;126:400-5. https://doi.org/10.1309/rrqkt2jeyv33d19d. 
9. Poller L, Ibrahim S, Keown M, Pattison A, Jespersen J, European Action on Anticoagulation. The prothrombin time/internationalnormalized ratio (PT/INR) Line: derivation of local INR with commercial thromboplastins and coagulometers two independent studies. J Thromb Haemost 2011;9:140-8. https://doi.org/10.1111/j.1538-7836.2010.04109.x.

10. Poller L. International normalised ratios (INR) - the first 20 years. J Thromb Haemost 2004;2:849-60. https://doi. org/10.1111/j.1538-7836.2004.00775.x.

11. Clinical and Laboratory Standards Institute (CLSI). Onestage prothrombin time (PT) and activated partial thromboplastin time (APTT) test; approved guideline H47-A2, 2nd ed. Wayne, PA: CLSI; 2008.

12. Fairweather RB, Ansell J, van den Besselaar AM, Brandt JT, Bussey HI, Poller L, et al. College of American Pathologists Conference XXXI on laboratory monitoring of anticoagulant therapy: laboratory monitoring of oral anticoagulant therapy. Arch Pathol Lab Med 1998;122:768-81.

13. Yim JM, Albers GW, Vlasses PH. Anticoagulant therapy monitoring with international normalized ratio at US academic health centers. Ann Pharmacother 1996;30:1390-5.

14. Howanitz PJ, Darcy TP, Meier FA, Bashleben CP. Assessing clinical laboratory quality. A College of American Pathologists Q-Probes Study on Prothrombin Time INR Structures, Processes and Outcomes in 98 Laboratories. Arch Pathol Lab Med 2015;139:1108-14. https://doi.org/10.5858/arpa.20140464-CP.

15. CCMB. [Harmonizacijala boratorijskih nalaza u području opće medicinske biokemije.] Available at: http://www. hkmb.hr/obavijesti/obavijesti-index.html. Accessed March 21th 2016. (in Croatian).

16. Pai M. Moffat KA, Plumhopff E, Hayward CPM. Critical values in coagulation laboratory: result on a survey of North American Specialized Coagulation Laboratory Association. Am J Clin Path 2011;136:836-41. https://doi.org/10.1309/ AJCP808GIPPPNUSH.

17. Brigden ML, Johnston M. A survey of aPTT reporting in Canadian medical laboratories. The need for increased standardization. Am J Clin Pathol 2000;114:276-82. https://doi. org/10.1309/4WK2-99F2-VEMB-15YJ.

18. Baglin T, Barrowcliffe TW, Cohen A, Greaves M. Guidelines on the use and monitoring of heparin. Br Soc Haematol 2006;133:19-34. https://doi.org/10.1111/j.13652141.2005.05953.x.

19. Marlar RA, Gausman J. The optimum number and types of plasma samples necessary for an accurate activated partial thromboplastin time - based heparin therapeutic range. Arch Pathol Lab Med 2013;137:77-82. https://doi. org/10.5858/arpa.2011-0516-OA.

20. Laposata M, Green D, Van Cott EM, Barrowcliffe TW, Goodnight SH, Sosolik RC. College of American Pathologists Conference XXXI on laboratory monitoring of anticoagulant therapy; the clinical use and laboratory monitoring of low-molecular-weight heparin, danaparoid, hirudin and related compounds, andargatroban. Arch Pathol Lab Med 1998; 122:799-807.

21. SahudM. Laboratory diagnosis of inhibitors. Semin Thromb Hemost 2000;26:195-203. https://doi.org/10.1055/s-2000-9823.
22. Pengo V. Tripodi A, ReberG, Rand H, Ortel TL, Galli M, De Groot PG. Update of the guidelines for lupus anticoagulant detection. J Thromb Haemost 2009;7:1737-40. https://doi. org/10.1111/j.1538-7836.2009.03555.x.

23. Kinra $P$, Tewari $V$, Raman RTS. Role of bleeding time and clotting time in preoperative hemostasis evaluation. Ind $J$ Aerospace Med 2009;53:56-61.

24. Chandler WL. Initial evaluation of hemostasis: reagent and method selection. In: Kitchen S, Olson JD, Preston E. Quality in laboratory hemostasis and thrombosis, 1st ed. Oxford: Wiley-Blackwell Publishing; 2009. p.63-71. https://doi. org/10.1002/9781444303575.ch7.

25. Chitolie A, Mackie IJ, Grant D, Hamilton JL, Machin $S M$. Inaccuracy of the "derived" fibrinogen measurement. Blood Coagul Fibrinol 1994;5:955-7. https://doi. org/10.1097/00001721-199412000-00012.

26. Miesbach W, Schenk J, Alesci S, Lindhoff-Last E. Comparison of the fibrinogen Clauss assay and the fibrinogen PT derived method in patients with dysfibrinogenemia. Thromb Res 2010;126:428-33. https://doi.org/10.1016/j.thromres.2010. 09.004.

27. Lawrie AS, McDonald SJ, Purdy G, Mackie IJ, Machin SJ. Prothrombin time derived fibrinogen determination on Sysmex CA-6000. J Clin Pathol 1998;51:462-6. https://doi. org/10.1136/jcp.51.6.462.

28. Mackie IJ, Lawrie AS, Kitchen S, Gaffney PJ, Howarth D, Lowe $G D O$, et al. A performance evaluation of commercial fibrinogen reference preparations and assays for Clauss and PTderived fibrinogen. Thromb Haemost 2002;87:997-1005.

29. Mackie IJ, Kitchen S, Machin SJ, Lowe GDO.On Behalf of the Haemostasis and Thrombosis Task Force of the British Committee for Standards in Haematology. Guidelines on fibrinogen assays. Br J Haematol 2003;121:396-404. https://doi. org/10.1046/j.1365-2141.2003.04256.x.

30. Douma RA, Tan M, Schutgens RE, Bates SM, Perrier A, Legnani $C$, et al. Using an age-dependent $D$-dimer cut-off value increases the number of older patients in whom deep vein thrombosis can be safely excluded. Haematologica 2012;97:1507-13. https://doi.org/10.3324/haematol.2011.060657.

31. Lippi G, Tripodi A, Simundic AM, Favaloro EJ. International survey on D-dimer test reporting: a call for standardization. Semin Thromb Hemost 2015;41:287-93. https://doi. org/10.1055/s-0035-1549092.

32. Olson JD, Cunningham MT, Higgins RA, Eby CS, Brandt JT. D-dimer: simple test, tough problems. Arch Pathol Lab Med 2013;137:1030-8. https://doi.org/10.5858/arpa.2012-0296$C P$.

33. Elferink RFM, Loot $A E$, Van de Klashorst CGJ, HulsebosHuygen M, Piersma-Wichers M, Oudega R. Clinical evaluation of eight different $D$-dimer test for the exclusion of deep venous thrombosis in primary care patients. Scand J Clin Lab Invest 2015;1-9. https://doi.org/10.3109/00365513.201 4.993697.

34. Favaloro EJ, Lippi G. New oral anticoagulants and the future of haemostasis laboratory testing. Biochem Med (Zagreb) 2012;22:329-41. https://doi.org/10.11613/BM.2012.035. 
35. Baglin T. The role of the laboratory in treatment with new oral anticoagulants. J Thromb Haemost 2013;11:122-8. https://doi.org/10.1111/jth.12227.

36. CCMB. [Pravilnici Hrvatske Komore medicinskih biokemičara. Pravilnik o načinu obavljanja medicinsko-biokemijske djelatnosti uz bolesnika.] Available at: http://www.hkmb. hr/opci-akti/dokumenti/HKMB-pravilnik-o-MBD-uz-bolesnika-2003.htm. Accessed March 21st 2016. (in Croatian).

37. Briggs C, Guthrie D, Hyde K, Mackie I, Parker N, Popek M, Porter N. Guidelines for point-of-care testing: haematology. Br J Haematol 2008;142:904-15. https://doi.org/10.1111/ j.1365-2141.2008.07274.x.

38. Perry DJ, Fitzmaurice DA, Kitchen S, Mackie IJ, Mallett S. Pointof-care testing in haemostasis. Br J Haematol 2010;150:50114. https://doi.org/10.1111/j.1365-2141.2010.08223.x.

39. Gardiner C, Williams K, Mackie IJ, Machin SJ, Cohen H. Patient self-testing is a reliable and acceptable alternative to laboratory INR monitoring. Br J Haematol 2004;128:242-7. https://doi.org/10.1111/j.1365-2141.2004.05300.x.
40. Heneghan C, Alonso-Coello P, Garcia-Alamino JM, Pere ra $R$, Meats $E$, Glasziou P. Self-monitoring of oral anticoagulation: a systematic review and meta-analysis. Lancet 2006;367:404-11. https://doi.org/10.1016/S0140-6736(06) 68139-7.

41. Bonar R, Favaloro EJ, Adcock DM. Quality in coagulation and haemostasis testing. Biochem Med (Zagreb) 2010;20(2):184-99. https://doi.org/10.11613/BM.2010.023.

42. Astermark J, Negrier $C$, Hermans $C$, Holme PA, Klamroth $R, P$. Kots $P$, et al. European Curriculum for Thrombosis \& Haemostasis developed by the European Association for Haemophilia and Allied Disorders (EHA). Haemophilia 2009;15:337-44. https://doi.org/10.1111/j.1365-2516.2008.01836.x.

43. CCMB. [Preporuke povjerenstva za stručna pitanja HKMB.] Available at: http://www.hkmb.hr/povjerenstva/strucna-pitanja.html\# 1. Accessed March 21st 2016. (in Croatian). 